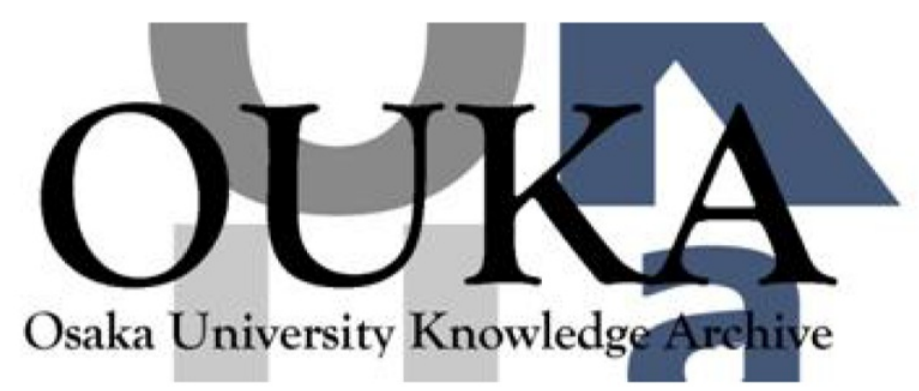

\begin{tabular}{|c|l|}
\hline Title & $\begin{array}{l}\text { Spin squeezing and decoherence Limit in Ramsey } \\
\text { spectroscopy }\end{array}$ \\
\hline Author(s) & Ulam-Orgikh, Duger; Kitagawa, Masahiro \\
\hline Citation & Physical Review A. 64(5) p.052106 \\
\hline Issue Date & $2001-10-08$ \\
\hline oaire:version & VoR \\
\hline URL & https://hdl.handle.net/11094/77655 \\
\hline rights & $\begin{array}{l}\text { Copyright (2001) by the American Physical } \\
\text { Society }\end{array}$ \\
\hline Note & \\
\hline
\end{tabular}

Osaka University Knowledge Archive : OUKA

https://ir. Library. osaka-u. ac. jp/

Osaka University 


\title{
Spin squeezing and decoherence limit in Ramsey spectroscopy
}

\author{
Duger Ulam-Orgikh* and Masahiro Kitagawa ${ }^{\dagger}$ \\ Graduate School of Engineering Science, Osaka University 1-3 Machikaneyama-cho, Toyonaka, Osaka 560-8531 Japan
}

(Received 31 March 2001; published 8 October 2001)

\begin{abstract}
It is known that a partially entangled state gives improved sensitivity in generalized Ramsey spectroscopy in the presence of decoherence, whereas a maximally entangled state does not. However, it has been an open question whether the known decoherence limit in the improvement is achievable. We show that every spinsqueezed state possesses pairwise entanglement, thereby improving the spectroscopic sensitivity, and that even suboptimal entanglement in its easiest implementation suffices to asymptotically reach the decoherence limit without any measurement optimization.
\end{abstract}

DOI: 10.1103/PhysRevA.64.052106

PACS number(s): 03.65.Ud, 03.65.Ta, 42.50.Dv, 03.67.-a

\section{INTRODUCTION}

While quantum correlations are still puzzling, they have already found many promising applications such as achieving interferometric [1-4] and spectroscopic [5,6] sensitivities beyond the standard quantum noise limit using squeezed states. Many more astonishing applications have appeared in a rapidly flourishing science of quantum information and computation [7], where entanglement serves as a basic ingredient. Both entanglement and squeezing are valuable resources of quantum correlations.

Entanglement is based on the superposition principle combined with Hilbert space structure, while squeezing is originated from another fundamental principle of quantum mechanics - the uncertainty principle. Therefore, it is interesting and important to study their relationship. Squeezed states of light (boson squeezing) have been well studied and used as an important source of entanglement in a theory of continuous variable quantum information (see Ref. [8] and references therein). A more advanced theory of discrete variable quantum information is based on a two-level quantum system or qubit. Thus, a concept of spin squeezing $[3-5,9,10]$ may be interesting and useful due to the spin-1/2 analogy of a qubit [11]. Unfortunately, spin squeezing is not a very well-understood issue and there are several definitions of it, which usually depend on the context in which they are being used [5,10,12-15].

A useful concept of spin squeezing in the sense of quantum correlations is established in Refs. $[3,9,10]$ and a terminology of a squeezed spin state (SSS) is introduced in contrast to a coherent spin state (CSS). Also, spin-squeezing models of one-axis twisting and two-axis twisting are proposed on the basis of nonlinear interaction between $N$ spins, which, respectively, reduce quantum noise down to the order of $N^{1 / 3}$ and 1/2. Lately, a variety of mechanisms of spin squeezing has been suggested utilizing the interaction of squeezed or nonclassical light with trapped atom/ions and metastable states of matter $[5,14,16,17]$, via quantum nondemolition measurement [18], in optical lattices [19] and some of the proposals have been experimentally demon-

\footnotetext{
*Electronic address: uka@laser.ee.es.osaka-u.ac.jp

${ }^{\dagger}$ Electronic address: kit@qc.ee.es.osaka-u.ac.jp
}

strated. A spin-squeezed ensemble of $10^{7}$ cold atoms has been experimentally observed [20] via absorption of nonclassical light. Squeezed spin states of atoms have been prepared by quantum nondemolition measurement on the collective spin of $10^{6}$ cesium atoms [21].

In the absence of decoherence, the squeezed uncertainty of a transverse spin component directly improves the sensitivity in interferometry [4] including Ramsey spectroscopy [5], enabling the improvement factors up to $N^{1 / 3}$ for one-axis twisting and $\sqrt{N}$ for two-axis twisting with $N$ particles [10]. Wineland et al. [5] have shown that a signal-to-noise ratio in the Ramsey spectroscopy may be increased by $\sim \sqrt{N}$ in certain cases over the case where uncorrelated $N$ are used, and possible experimental demonstrations are discussed. Another interesting scheme that improves interferometric sensitivity by a factor of $\sqrt{N}$ is possible by using maximally entangled states $[22,23]$ treating $N$ particles as a single-quantum object. The phase evolution of an object consisting of $N$ particles is $N$ times as fast as that of a particle itself, or an equivalently deBroglie wavelength is shortened by a factor of $N$ [22], giving the standard quantum limit for an object $N \delta \phi \sim 1$.

In the presence of decoherence, the second scheme will collapse very rapidly for large $N$ while the first one will partly survive [24]. For small $N$, an experiment using a stateof-the-art ion trap is suggested to be feasible [23] and entanglement of four ions have been indeed demonstrated [25]. The demonstration of the drastic improvement over the standard quantum limit is the ultimate experimental goal that requires the suppression of decoherence-a very challenging task. Another direction we may seek is to go along with decoherence and find the best we can do under decoherence. Huelga et al. [24] have shown that, in the presence of decoherence, a maximally entangled state does not provide higher resolution, whereas a partially entangled state with a high symmetry gives an improved sensitivity. A quantum network construction for such a highly symmetric state is given in [26].

But it has not been clear how this state should be entangled, and to what extent the sensitivity is improved. Moreover, the symmetry does not by itself guarantee that the state is optimal in the sense it gives maximal precision. This is because in generalized Ramsey spectroscopy, the sum of all spins are measured and the above-mentioned high degree of symmetry only relates to the signal strength, i.e., classical 
correlations among qubits, but it does not relate to the quantum noise, i.e., quantum correlations or entanglement among qubits. An optimization of the coefficients of the highly symmetric states is also a difficult problem for large $N$. Therefore, it has been an open problem whether a precision improvement saturates at the theoretical decoherence limit with increasing $N$ [24].

We answer these questions in this paper by showing that the decoherence limit is asymptotically achievable, even when the simplest spin-squeezed input state is used, which has nonoptimal but suboptimal entanglement.

The paper is organized as follows. In Sec. II, we demonstrate that a squeezed spin state possesses pairwise entanglement. In Sec. III, a scheme of generalized Ramsey spectroscopy with an input SSS is summarized. We show that an SSS always gives an improvement in precision in the presence of decoherence. In the final section, we prove that the improvement asymptotically reaches the theoretical decoherence limit even for a suboptimal input state generated by one-axis twisting. Throughout the paper, we refer to $\mathrm{N}$-cat states [27], $\left(|0\rangle^{\otimes N}+|1\rangle^{\otimes N}\right) / \sqrt{2}$, as maximally entangled in the sense of Einstein-Podolsky-Rosen correlation. It is shown that maximally entangled states are nonspin squeezed for $N \geqslant 3$ and completely spin squeezed for $N=2$.

\section{SPIN SQUEEZING AND PAIRWISE ENTANGLEMENT}

Before dealing with the main subject of the paper, we briefly discuss the relationships between spin squeezing and entanglement. Several definitions of spin squeezing are used in the literature $[5,10,12-15]$. A definition of spin squeezing based only on the uncertainty relation [12] is not invariant under rotation; thus, it does not necessarily reflect the quantum correlations. Therefore, we follow here a definition of spin squeezing based on quantum spin correlations as in Ref. [10], where spin was regarded as squeezed only if the minimum variance $\left\langle\Delta S_{\perp}^{2}\right\rangle$ of a spin component perpendicular to the direction $\hat{n}$ of the mean spin vector is smaller than the standard quantum limit N/4 of the CSS. Here, a collective spin operator for $N$ spin $1 / 2$ is defined as

$$
S_{k}=\frac{1}{2} \sum_{i=1}^{N} \sigma_{k, i} \quad(k=x, y, z),
$$

where $\sigma_{k, i}$ is a Pauli operator for $i$ th spin $1 / 2$. At this point, it is convenient to introduce the following quantities normalized with respect to the CSS:

$$
\begin{gathered}
v_{\perp}=\frac{4}{N}\left\langle\Delta S_{\perp}^{2}\right\rangle, \quad\left(v_{\perp} \leqslant 1\right), \\
\eta_{\hat{n}}=\frac{4}{N^{2}}\left\langle S_{\hat{n}}\right\rangle^{2} \quad\left(\eta_{\hat{n}} \leqslant 1\right) .
\end{gathered}
$$

Then, $v_{\perp}$ serves as a squeezing parameter and our definition of spin squeezing simply reads

$$
v_{\perp}<1 .
$$

The spin squeezing in the original sense [10] is well defined only within the maximum $2 S+1(S=N / 2)$ multiplicity subspace to which the CSS belongs, since it is defined with respect to the standard quantum limit of the CSS. In other words, this definition makes sense only for the eigenstates of $\hat{S}^{2}$ with the maximal eigenvalue. For instance, we may say about spin squeezing of triplet states, but not about the singlet one when $N=2$. As for another example, if one starts from an initial CSS and applies only collective operators $f\left(S_{x}, S_{y}, S_{z}\right)$, then the state remains in the subspace of maximum multiplicity because of $\left[f\left(S_{z}, S_{y}, S_{z}\right), \hat{S}^{2}\right]=0$, and the concept of spin squeezing may be safely used. Clearly, the state may not be confined to this particular subspace in other circumstances, for instance, in the presence of decoherence as the case discussed in this paper. A further generalization of spin squeezing will be discussed elsewhere.

Negative spin correlations are implied by the above definition of spin-squeezing Eq. (4), as may be seen in the following way: If we define a pairwise correlation coefficient [15] (or statistical covariance) as $C_{k k^{\prime}}^{i j}=1 / 4\left(\left\langle\sigma_{k, i} \sigma_{k^{\prime}, j}\right\rangle\right.$ $\left.-\left\langle\sigma_{k, i}\right\rangle\left\langle\sigma_{k^{\prime}, j}\right\rangle\right)\left(k, k^{\prime}=x, y, z\right)$, then it is easy to show that the condition for negative correlations may be written in the form $C_{k k}^{i j}=\left(v_{k}+\eta_{k}-1\right) /[4(N-1)]<0$ or simply $v_{k} /(1$ $\left.-\eta_{k}\right)<1$. In the principal coordinate $(\hat{n}, \perp)$, it reads $v_{\perp}<1$, since $\eta_{\perp}=0$ by the definition. Thus, the definition of SSS given by Eq. (4) is equivalent to the condition for negative pairwise correlations.

It is more important that these correlations are quantum in nature in the maximum multiplicity subspace, for which the definition of spin squeezing is supposed to be used. This may be understood intuitively as follows: For a state of pair qubits in the triplet sub-space $|\Psi\rangle=\alpha|00\rangle+\beta(|01\rangle$ $+|10\rangle) / \sqrt{2}+\gamma|11\rangle$, where $\alpha, \beta$, and $\gamma$ are arbitrary constants, one may find that the concurrence $C$-a measure of entanglement [28] has one-to-one correspondence with $v_{\perp}$ as

$$
C=\left|2 \alpha \gamma-\beta^{2}\right|=1-v_{\perp} .
$$

It implies that a spin-squeezed pair spins is always entangled or quantum correlated. On the other hand, a state in the maximum multiplicity subspace has a Clebsch-Gordan composition of separated single spins and entangled pair spins. Therefore, pairwise negative correlations in Eq. (4) are quantum, i.e., an SSS always possesses pairwise entanglement. A more detailed discussion on this subject will be published elsewhere.

Another implication is that a $N$-cat maximally entangled state is nonspin squeezed, i.e., it has no pairwise or bipartite entanglement, since it is truly $N$-partite entangled [29]. Our result is in full agreement with the fact that the pairwise entanglement is the least fragile under the disposal one of the qubits (spins), while maximally entangled states are the most fragile ones having genuine $N$-partite entanglement. We note that CSS is the only separable state in the maximum multiplicity subspace and the spin-squeezing condition Eq. (4) is a sufficient (but not necessary) condition for nonseparability.

This can alternatively be shown by the same technique as in Ref. [30], where another (more strict) sufficient condition 
for inseparability was given. Namely, $v_{\hat{n}_{1}} /\left(\eta_{\hat{n}_{2}}+\eta_{\hat{n}_{3}}\right) \leqslant 1$, here $\hat{n}_{1}, \hat{n}_{2}$, and $\hat{n}_{3}$ are orthogonal directions. This condition is closely connected with another widely used definition of spin-squeezing $v_{\perp} / \eta_{\hat{n}}<1$, which indicates a level of spectroscopic precision improvement in the absence of decoherence [5], since $\eta_{\hat{n}_{2}}+\eta_{\hat{n}_{3}}<1$. But, it is undetermined for the maximally entangled state, thus no longer useful at least for the purpose of this paper. We just note that our definition is equivalent whenever the spectroscopic one is valid (in particular for small squeezing).

The pairwise entanglement of SSS, being quantum negative correlations, improves the measurement sensitivity in Ramsey spectroscopy in the presence of decoherence, and we show this in the next section.

\section{GENERALIZED RAMSEY SPECTROSCOPY SCHEME}

The Nobel prize-winning Ramsey spectroscopy method is well known (see [14,31] for details). Since the purpose of the paper is to answer the open question given in Ref. [24], where a generalized Ramsey spectroscopy scheme is discussed, we consider here the same scheme but with a spinsqueezed input state. We note that this scheme has the following simplifications; the decoherence effect is considered only during the free-evolution period of Ramsey spectroscopy but not for input state preparation, and a period of input state preparation is not considered. The problem for more realistic situations will be discussed separately.

Suppose that we are given $N$ ions in a trap. The generalized Ramsey spectroscopy scheme starts by applying the first Ramsey pulse $R_{y}(\pi / 2)=\exp \left(-i \pi S_{y} / 2\right)$ to an initial CSS $|0\rangle^{\otimes N}$, which rotates the CSS to point in the $x$ direction. This is then followed by spin-squeezing unitary evolution $\exp \left(-i H_{s s}\right) t / \hbar$ with squeezing Hamiltonian $H_{s s}$. Under the spin-squeezing evolution, the maximum squeezing axis $\perp$ is altered, while the main spin direction $\hat{n}$ is not changed, as we see later. Thus, another rotation pulse $R_{x}(\nu)=\exp \left(-i \nu S_{x}\right)$ is needed to direct the maximal squeezing in a desired direction, namely along the $y$ axis. (Or, the initial CSS may be squeezed before the first Ramsey pulse is applied.) Therefore, an input SSS is prepared as

$$
\left|\Psi_{s s}\right\rangle=R_{x}(-\nu) \exp \left(-i H_{s s} t / \hbar\right) R_{y}\left(\frac{\pi}{2}\right)|0\rangle^{\otimes N} .
$$

Following this, the state undergoes a period of "free evolution" during which we assume a decoherence is present. Each spin is assumed to evolve independently via the master equation $\rho \dot{(t}=-i[H, \rho]-\gamma(\rho-1 / 2)$, i.e., nondiagonal elements are decayed with a rate $\gamma$ [24]. A different model of decoherence is discussed in Ref. [32]. Finally, the collective operator $S_{y}$ is measured with the use of the second Ramsey pulse. This basic scheme is repeated many times for a total experiment duration $T$.

The uncertainty in atomic frequency according to this scheme is defined by

$$
\left|\delta \omega_{0}\right|_{s s}=\sqrt{\left(1 / N_{m}\right)\left[\left(\Delta S_{y}\right)^{2} /\left(\partial\left\langle S_{y}\right\rangle / \partial \omega\right)^{2}\right]},
$$

where $N_{m}$ denotes the total number of the repeated measurements. A relative improvement in the precision over the minimal uncertainty $\left|\delta \omega_{0}\right|=\sqrt{2 e \gamma / N T}$ for the standard Ramsey spectroscopy with an uncorrelated input state [24] may be found in the form

$$
P\left(\tau, v_{y}, \eta_{x}\right)=1-\frac{\left|\delta \omega_{0}\right|_{s s}}{\left|\delta \omega_{0}\right|}=1-\sqrt{\frac{e^{\tau}-1+v_{y}}{e \eta_{x} \tau}},
$$

where $\tau=2 \gamma t_{f}$ and $t_{f}$ is a free-evolution time for single step. It is easy to analyze Eq. (7) if we remember that $v_{y}$ and $\eta_{x}$ are normalized with respect to the CSS to be $v_{y} \leqslant 1$ and $\eta_{x}$ $\leqslant 1$. We note that this equation has a singularity in the limit $\tau \rightarrow 0$, unless also $v_{y} \rightarrow 0$.

If we consider Eq. (7) as a function on $\tau$, then its form is defined by $v_{y}$ : it is a convex function with the maximum $P_{\max }>0$ when $v_{y}<1$, and a monotonically decreasing function $P \leqslant 0$ when $v_{y}=1$. In other words, all SSS's possess the potential for improving precision in the presence of decoherence, while non-SSS's such as uncorrelated or maximally entangled states, do not give any improvement according to this generalized Ramsey spectroscopy scheme for $t_{f}>\tau$ [33].

Since spin squeezing occurs in a spherical angular momentum phase space, not only $v_{\perp}$ but also $\eta_{\hat{n}}$ is decreased with an increasing of squeezing. The former gives an improvement while the latter degrades it according to Eq. (7). The competition between them under the decoherence process justifies the actual maximal improvement and one does not necessarily need the maximal spin squeezing for the best precision. That explains why a simple spin squeezing model of one-axis twisting turns out to be useful.

When we neglect the pulse duration and squeezing period, the free-evolution time $t$ equals to a single measurement time, which actually needs to be optimized for the maximal improvement. By optimizing Eq. (7) on $\tau$, one sees that the optimal value $\tau_{\mathrm{opt}}$ is determined by the equation $\tau_{\mathrm{opt}}=1$

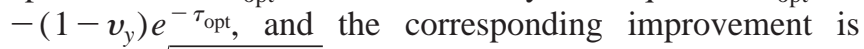

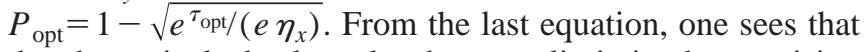
the theoretical absolute decoherence limit in the precision improvement is

$$
P_{\mathrm{abs}}=1-\frac{1}{\sqrt{e}} \simeq 0.39 \quad\left(\text { when } \tau_{\mathrm{opt}} \rightarrow 0, \quad \eta_{x} \rightarrow 1\right) .
$$

The full optimization of Eq. (7) on $\eta_{x}$ and $v_{y}$ functions is not a simple problem especially for large $N$, for instance; a full numerical optimization with respect to the input state preparation have been done only up to $N=7$ in Ref. [24]. Thus, it was not clear whether the decoherence limit is reachable by increasing the number of ions [24,34], and we answer this question in the next section.

\section{SSS AND DECOHERENCE LIMIT}

In this section, using a one-axis twisting model of spin squeezing we show that the decoherence limit in the improvement of spectroscopic precision may be reached asymptotically. A nonlinear Hamiltonian of one-axis twisting is defined as 


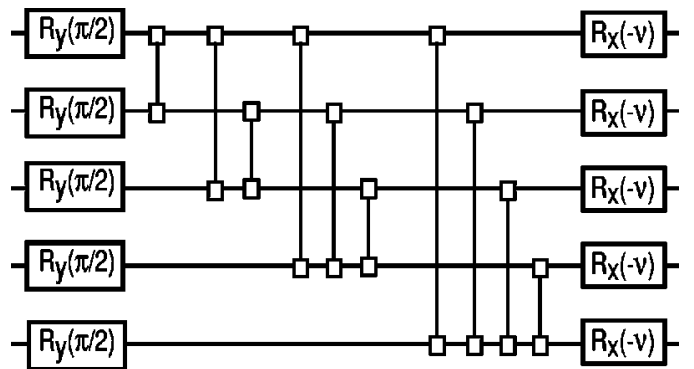

FIG. 1. Efficient quantum network for preparation of an initial SSS. The two-qubit symmetric gate is a controlled rotation gate defined by the unitary operator of $\exp \left[-i \mu \sigma_{z, i} \sigma_{z, j} / 8\right]$, i.e., a rotation $R_{z}^{(i j)}(\mp \mu / 4)$ is applied to the second qubit depending on the value, 0 or 1 , of the first qubit or vice versa.

$$
H_{s s}=\hbar \chi S_{z}^{2},
$$

where $S_{z}=1 / 2 \sum_{i=1}^{N} \sigma_{z, i}$. This model is quite general and may be realized experimentally, for example, by illuminating all the ions in a trap with laser beams with two different colors [35] or one deals with a similar Hamiltonian in a two-mode approximation of a many-body Hamiltonian in neutral atomic Bose-Einstein condensate in a double-well potential [36]. A conditional displacement of the vibrational mode of trapped ions may also be used to simulate such a nonlinear Hamiltonian [37].

The one-axis twisting evolution may also be simulated by an efficient quantum network, which is easy to see by writing the unitary evolution operator in the form

$$
U_{s s}=\exp \left(-i \chi S_{z}^{2} t\right)=\prod_{i<j} \exp \left(-i \frac{\mu}{8} \sigma_{z, i} \sigma_{z, j} t\right),
$$

where $\mu=2 \chi t$ is a spin-squeezing angle and a constant term is neglected. The two-spin entangling evolution exp $\left(-i(\mu / 8) \sigma_{z, i} \sigma_{z, j}\right)$ may be easily implemented via a symmetrically controlled rotation gate, that is, a rotation gate

$$
R_{z}^{(i j)}\left(\mp \frac{\mu}{4}\right)=\left(\begin{array}{cc}
e^{\mp i \mu / 8} & 0 \\
0 & e^{ \pm i \mu / 8}
\end{array}\right)
$$

is applied to the second qubit depending on the value, 0 or 1 , of the first qubit. Thus, the preparation of the input SSS may be expressed as an efficient [38] quantum network as shown in Fig. 1, consisting of $2 N$ one-qubit and $N(N-1) / 2$ twoqubit gates. form

Using Eqs. (9) and (6), one finds the input state in the

$$
\left|\Psi_{s s}\right\rangle=\sum_{l=0}^{N} c_{l}(\mu, \nu)\left|\varphi_{l}^{\mathrm{perm}}\right\rangle
$$

where $\left|\varphi_{l}^{\text {perm }}\right\rangle=\Sigma_{\text {perm }}\left|1^{\otimes l} 0^{\otimes(N-l)}\right\rangle$ is a permutation symmetric state with $l$ being the number of 1 's and the coefficient is

$$
\begin{aligned}
c_{l}(\mu, \nu)= & 2^{-N / 2} \sum_{k=0}^{l} \sum_{m=k}^{N} \exp \left[-i \frac{\mu}{2}(m-N / 2)^{2}\right]\left(\begin{array}{l}
l \\
k
\end{array}\right)\left(\begin{array}{c}
N-l \\
m-k
\end{array}\right) \\
& \times\left(\cos \frac{\nu}{2}\right)^{l+m-2 k}\left(i \sin \frac{\nu}{2}\right)^{N-l-m+2 k}
\end{aligned}
$$

The state $\left|\Psi_{s s}\right\rangle$ is also symmetric under the exchange of the excited and ground states for each ion, since $c_{l}(\mu, \nu)$ $=c_{N-l}(\mu, \nu)$. It should be noted that in generalized Ramsey spectroscopy we have to deal, indeed, with high-symmetric states as a natural consequence of the following two facts: (1) Only the sum of spin (dipole) components is measured in Ramsey spectroscopy and any permutation among qubits does not change the result, therefore, the state has a permutation symmetry. (2) Only the relative phase between $|0\rangle$ and $|1\rangle$ is measured, the mean spin vector must be in the $x-y$ plane to maximize the signal, and $|0\rangle$ and $|1\rangle$ have the same weight in each qubit, i.e., a symmetry under exchange of $|0\rangle$ and $|1\rangle$.

For discussing spin squeezing, we need to find $\eta_{k}$ and $v_{k}$, which are given [10] by

$$
\begin{gathered}
\eta_{x}(\mu)=\eta_{n}=\cos ^{2(N-1)} \frac{\mu}{2}, \quad \eta_{y}=\eta_{z}=0, \\
v_{y}(\mu)=v_{\perp}=1-\frac{1}{4}(N-1)\left[\sqrt{A^{2}+B^{2}}-A\right],
\end{gathered}
$$

where $A=1-\cos ^{N-2} \mu$ and $B=4 \sin (\mu / 2) \cos ^{N-2}(\mu / 2)$. As one can see, under one-axis twisting, the main spin direction does not change. Therefore, $v_{y}$ is chosen to be minimal in Eq. (15) by applying the adjusting pulse $R_{x}(\nu)$ with $\nu$ being determined via $\mu$ as $\nu=\pi / 2-1 / 2 \arctan B / A$. We note that the coefficient $c_{l}$ of Eq. (13) actually depends on the only one parameter $\mu$ when $v_{\perp}=v_{y}$.

From Eq. (15) it can be seen that the states are spinsqueezed $v_{y}(\mu)<1$ when $0<\mu<\pi$, while at $\mu=\pi$, they become nonspin squeezed as $v_{y}(\pi)=1$ for $N \geqslant 3$. On the other hand, from Eq. (13) it follows that the states at $\mu=\pi$ are maximally entangled states, for instance, they are in $N$-cat form: $c_{0}(\pi, \pi / 2)=c_{N}(\pi, \pi / 2)=1 / \sqrt{2}$ for $N_{\text {odd }}$. The maximality of entanglement for $N_{\text {even }} \geqslant 4$ spins may be seen in the following way [39]: it may be written in $N$-cat state form $1 / \sqrt{2}\left(|0\rangle^{\otimes N}+|1\rangle^{\otimes N}\right)$, as for $N_{\text {odd }}$ case, after using a rotation pulse $R_{y}(\pi / 2)$ for all spins, which do not change the entanglement. Therefore, we have seen that a maximally entangled state is non-SSS for $N \geqslant 3$. In contrast, the maximally entangled two-qubit states (with $\nu=3 \pi / 4$ and $\nu=\pi / 4$ ) are completely spin-squeezed ones $v_{y}(\pi)=0$, as expected.

Finally, let us return to the precision improvement problem again, since now we have all necessary information and analytic expressions. For one-axis twisting, the optimization of Eq. (7) for the maximal improvement is done on only two parameters, $\mu$ and $\tau$, regardless of the number of ions, instead of $\lfloor N / 2\rfloor+1$ ones for numerical optimization of the input state [24], thus drastically simplifying the problem and allowing full analysis, but at the cost of suboptimality of the input state. 

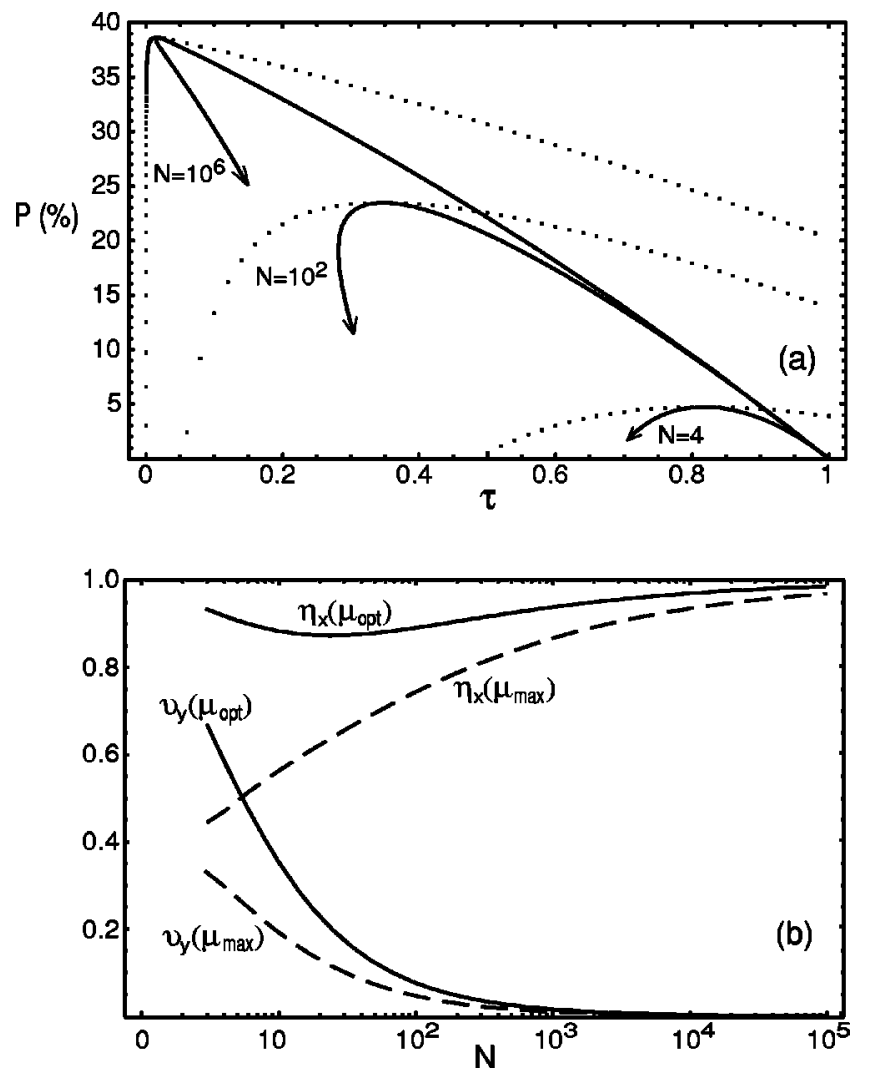

FIG. 2. Optimization and usefulness of one-axis twisting. (a) Optimization of $P(\tau, \mu)$, where $\tau=2 \gamma t_{f}, t_{f}$ is a free-evolution time for single step, $\gamma$ is a decoherence rate, and $\mu$ is the squeezing angle; solid line: $P\left(\tau_{\mathrm{opt}}, \mu\right)$. Arrow is an increasing direction of $\mu$ and its explicit value is not shown. Dotted line: $P\left(\tau, \mu_{\text {opt }}\right)$. Intersection of these two curves is the maximal improvement in the precision $P_{\max }$ for a given number of ions $N$; (b) normalized variance $v_{y}$ (squeezing parameter) and square of the mean $\eta_{x}$ vs $N$; the index opt is for optimal spin squeezing, which gives the best precision and the index $\max _{\text {ax }}$ is for available maximal spin squeezing.

As we have discussed in the previous section, $v_{y}(\mu)$ as well as $\eta_{x}(\mu)$ decrease with an increasing of squeezing but at a different rate, and the actual maximal improvement is resolved by their competition via Eq. (7). It is interesting to see how $P(\tau, \mu)$ is actually optimized via the two optimization parameters. In Fig. 2(a), we have plotted $P\left(\tau_{\text {opt }}, \mu\right)$ versus $\tau_{\text {opt }}$ and $P\left(\tau, \mu_{\text {opt }}\right)$ versus $\tau$ for a given $N$, in other words, by fixing one of the variables as a parameter and optimizing on the other one. As can be seen from $P\left(\tau_{\text {opt }}, \mu\right)$, small squeezing always gives an improvement, which degrades for further squeezing due to it being more sensitive to change in $\eta_{x}(\mu)$ near the optimal squeezing. As a result, maximal improvement occurs for relatively small $\mu$ and $\eta_{x}\left(\mu_{\text {opt }}\right)$ is reduced very slightly [see Fig. 2(b)].

Therefore, the best precision in the presence of decoherence does not require maximal spin squeezing, which explains why a simple model of spin squeezing, such as oneaxis twisting, turns out to be useful. The $P\left(\tau_{\text {opt }}, \mu\right)$ dependence becomes complicated for large $N$, due to the appearance of an instability near the optimal solution for the best precision. This indicates another very noticeable-not
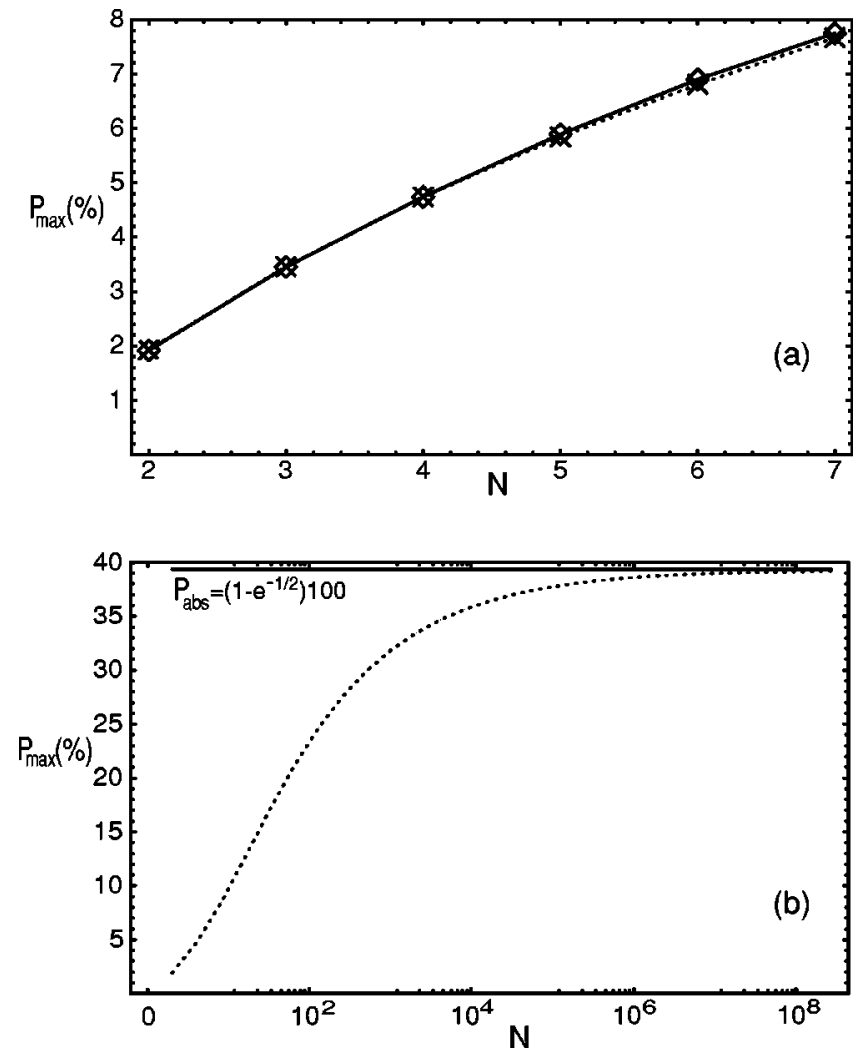

FIG. 3. Maximal improvement $P_{\max }$ vs number of ions $N$. (a) Comparison for the optimal and spin squeezed (suboptimal) input states; $\diamond$, full numerical optimization of the initial state preparation [24]; $\times$, initial SSS preparation via one-axis twisting; (b) the absolute decoherence limit $1-1 / \sqrt{e}$ (solid line) is asymptotically reached even for suboptimal entanglement (dotted line) of one-axis twisting.

explicit from Eq. (7)-difficulty in finding the optimal input state.

In Fig. 3, the maximal improvement of Eq. (7) is plotted versus the number of ions $N$. One sees that the one-axis twisting is a good approximation to the optimal solution, at least for the small number of ions as in Fig. 3(a). The improvement in the precision asymptotically reaches at the absolute decoherence limit with increasing $N$ in Fig. 3(b), and it is obvious that a more sophisticated model, such as the two-axis twisting, gives quicker saturation. As a matter of fact, for large number of $N$, from Eqs. (14)-(15), we have [10]

$$
v_{\perp} \simeq \frac{1}{4 \alpha}+\frac{2}{3} \beta^{2} \quad \text { and } \quad \eta_{\hat{n}} \simeq(1-\beta)^{2}
$$

when $\mu \ll 1$, provided $\alpha=(N / 4) \mu \gg 1$ and $\beta=(N / 8) \mu^{2} \ll 1$. The latter conditions are in good agreement for $N>10^{5}$. When $\tau \ll 1$, from Eq. (7), we have $P \simeq 1-(1 / \sqrt{e})\{1$ $\left.+d N^{-1 / 3}\right\}$, where $d=\left[(1 / 6)^{1 / 6}+(3 / 4)^{1 / 3}\right] \simeq 1.6504$, thus,

$$
\lim _{N \rightarrow \infty} P=1-\frac{1}{\sqrt{e}}=P_{a b s} .
$$


It is a little surprising that the theoretical decoherence limit Eq. (8) is asymptotically reached even in the case of suboptimal entanglement of a simple SSS-one does not need a full optimization of the input state nor measurement optimization. Decoherence washes out the details of entanglement or squeezing.

\section{CONCLUSION}

We have considered a generalized Ramsey spectroscopy scheme with a spin-squeezed input state and shown that a SSS is a useful resource of entanglement, intrinsically possessing an ability for improving spectroscopic precision in the presence of decoherence due to its negative pair-wise quantum correlations. This also explains why nonspinsqueezed states, such as a maximally entangled state, do not give any improvement in that situation. As the main result of the paper, we have answered the open question [24], by showing that the improvement in precision asymptotically saturates at the theoretical decoherence limit Eq. (8) with an increasing number of ions; even suboptimal entanglement of a simple spin squeezing is enough for it, i.e., neither full optimization of input state nor measurement optimization is required.

\section{ACKNOWLEDGMENTS}

D.U. gratefully acknowledges the financial support of the Japanese Ministry of Education (Monbusho) and of CREST, Japan Science and Technology (JST). We are grateful to Professor M. Ueda for valuable discussions. D.U. thanks Dr. M. W. Jack for useful comments.
[1] C.M. Caves, Phys. Rev. D 23, 1693 (1981).

[2] B. Yurke, Phys. Rev. Lett. 56, 1515 (1986).

[3] M. Kitagawa and M. Ueda, in The International Conference on Noise in Physical Systems and 1/f Fluctuations, Kyoto, Japan, 1991, edited by T. Musha, S. Sato, and Y. Yamamoto (Omsha, Tokyo, 1991), pp. 355-358.

[4] M. Kitagawa and M. Ueda, Phys. Rev. Lett. 67, 1852 (1991).

[5] D.J. Wineland, J.J. Bollinger, W.M. Itano, F.L. Moore, and D.J. Heinzen, Phys. Rev. A 46, R6797 (1992).

[6] W.M. Itano, J.C. Bergquist, J.J. Bollinger, J.M. Gilligan, D.J. Heinzen, F.L. Moore, M.J. Raizen, and D.J. Wineland, Phys. Rev. A 47, 3554 (1993).

[7] H.K. Lo, S. Popesku, and T. Spiller, Introduction to Quantum Computation and Information (World Scientific, Singapore, 1997).

[8] S.F. Pereira, Z.Y. Ou, and H.J. Kimble, Phys. Rev. A 62, 042311 (2000).

[9] For comparison of boson and spin squeezing, see M. Kitagawa and M. Ueda, in 2nd International Workshop on Squeezed States and Uncertainty Relations, Moscow, 1992, edited by D. Han, Y.S. Kim, and V.I. Man'ko, NASA Conference Publications No. 3219 (U.S. GPO, Washington, DC, 1993), pp. 187192.

[10] M. Kitagawa and M. Ueda, Phys. Rev. A 47, 5138 (1993).

[11] R.P. Feynman, F.L. Vernon, Jr., and R.W. Hellwarth, J. Appl. Phys. 28, 49 (1957).

[12] K. Wódkiewicz and J.H. Eberly, J. Opt. Soc. Am. B 2, 458 (1985)

[13] G.S. Agarwal and R.R. Puri, Phys. Rev. A 41, 3782 (1990).

[14] D.J. Wineland, J.J. Bollinger, W.M. Itano, and D.J. Heinzen, Phys. Rev. A 50, 67 (1994).

[15] G.S. Agarwal and R.R. Puri, Phys. Rev. A 49, 4968 (1994).

[16] A. Kuzmich, K. Molmer, and E.S. Polzik, Phys. Rev. Lett. 79, 4782 (1997).

[17] M.D. Lukin, S.F. Yelin, and M. Fleischhauer, Phys. Rev. Lett. 84, 4232 (2000).

[18] A. Kuzmich, N.P. Bigelow, and L. Mandel, Europhys. Lett. 42, 481 (1998).

[19] A. Sorensen and K. Molmer, Phys. Rev. Lett. 83, 2274 (1999).
[20] J. Hald, J.L. Sorensen, C. Schori, and E.S. Polzik, Phys. Rev. Lett. 83, 1319 (1999).

[21] A. Kuzmich, L. Mandel, J. Janis, Y.E. Young, R. Ejnisman, and N.P. Bigelow, Phys. Rev. A 60, 2346 (1999).

[22] J. Jacobson, G. Björk, I. Chuang, and Y. Yamamoto, Phys. Rev. Lett. 74, 4835 (1995).

[23] J.J. Bollinger, W.M. Itano, D.J. Wineland, and D.J. Heinzen, Phys. Rev. A 54, R4649 (1996).

[24] S.F. Huelga, C. Macchiavello, T. Pellizzari, A.K. Ekert, M.B. Plenio, and J.I. Cirac, Phys. Rev. Lett. 79, 3865 (1997).

[25] C.A. Sackett, D. Kielpinski, B.E. King, C. Langer, V. Meyer, C.J. Myatt, M. Rowe, Q.A. Turchette, W.M. Itano, D.J. Wineland, and C. Monroe, Nature (London) 404, 256 (2000).

[26] H. Azuma, Int. J. Mod. Phys. C 11(3), 469 (2000).

[27] C.H. Bennett, S. Popescu, D. Rohrlich, J.A. Smolin, and A.V. Thapliyal, e-print quant-ph/9908073.

[28] S. Hill and W.K. Wootters, Phys. Rev. Lett. 78, 5022 (1997).

[29] W. Dür, G. Vidal, and J.I. Cirac, e-print quant-ph/0005115.

[30] A. Sorensen, L.M. Duan, J.I. Cirac, and P. Zoller, e-print quant-ph/0006111.

[31] N. F. Ramsey, Molecular Beams (Oxford, London, 1963).

[32] A.M. Childs, J. Preskill, and J. Renes, e-print quant-ph/9904021.

[33] As suggested in Ref. [23], maximally entangled states might be useful for $t_{f}<\tau$, which is another regime of experimental interest.

[34] S.L. Braunstein and C.M. Caves, Phys. Rev. Lett. 72, 3439 (1994).

[35] K. Molmer and A. Sorensen, Phys. Rev. Lett. 82, 1835 (1999).

[36] G.J. Milburn, J. Corney, E.M. Wright, and D.F. Walls, Phys. Rev. A 55, 4318 (1997).

[37] G.J. Milburn, e-print quant-ph/9908037.

[38] A. Barenco, C.H. Bennett, R. Cleve, D.P. DiVincenzo, N. Margolus, P. Shor, T. Sleator, J.A. Smolin, and H. Weinfurter, Phys. Rev. A 52, 3457 (1995).

[39] M. Kitagawa, in CLEO/Pacific Rim'97, The Pacific Rim Conference on Lasers and Electro-Optics, Tech. Digest, IEEE Catalog No. 97TH8275, WG3, 1997, p. 80. 